
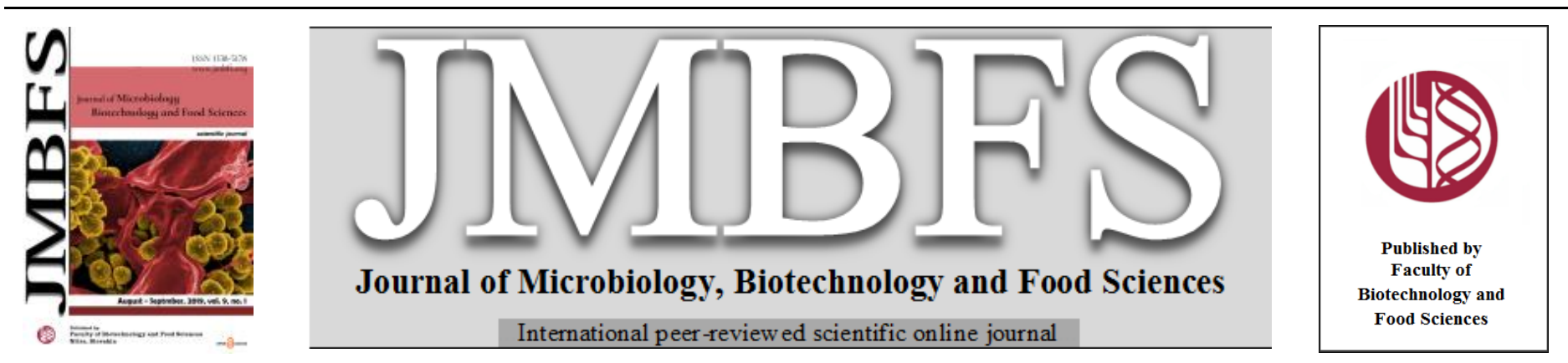

\title{
THE DIVERSITY OF CULTIVABLE HYDROCARBON-DEGRADING BACTERIA ISOLATED FROM CRUDE OIL CONTAMINATED SOIL AND SLUDGE FROM ARZEW REFINERY IN ALGERIA
}

\author{
Sonia SEKKOUR ${ }^{{ }^{*}}$, Abdelkader BEKKI ${ }^{l}$, Zoulikha BOUCHIBA ${ }^{1}$, Timothy M. Vogel ${ }^{2}$,Elisabeth NAVARRO ${ }^{2}$
}

Address(es): Ing. Sonia SEKKOUR PhD.,

${ }^{1}$ Université Ahmed Benbella, Faculté des sciences de la nature et de la vie, Département de Biotechnologie, Laboratoire de biotechnologie des rhizobiums et amélioration des plantes, 31000 Oran, Algérie.

${ }^{2}$ Environmental Microbial Genomics Group, Laboratoire Ampère, Centre National de la Recherche Scientifique, UMR5005, Institut National de la Recherche Agronomique, USC1407, Ecole Centrale de Lyon, Université de Lyon, Ecully, France.

*Corresponding author: sekkour.sonia@gmail.com

doi: $10.15414 /$ jmbfs.2019.9.1.70-77

ARTICLE INFO

Received 27.3. 2018

Revised 19. 2. 2019

Accepted 14.3. 2019

Published 1. 8. 2019

Regular article

open $\mathcal{O}_{\text {ACCESS }}$

\begin{abstract}
The use of autochtonious bacterial strains is a valuable bioremediation strategy for cleaning the environment from hydrocarbon pollutants. The isolation, selection and identification of hydrocarbon-degrading bacteria is therefore crucial for obtaining the most promising strains for decontaminate a specific site. In this study, two different media, a minimal medium supplemented with petroleum and with oil refinery sludge as sole carbon source, were used for the isolation of native hydrocarbon-degrading bacterial strains from crude oil contaminated soils and oil refinery sludges which allowed isolation of fifty-eight strains.The evalution of diversity of twentytwo bacterials isolates reveled a dominance of the phylum Proteobacteria (20/22 strains), with a unique class of Alphaproteobacteria, the two remaining strains belong to the phylum Actinobacteria. Partial 16S rRNA gene sequencing performed on isolates showed high level of identity with known sequences. Strains were affiliated to Sinorhizobium, Promicromonospora, Novosphingobium, Georgenia, Ancylobacter, Roseomonas, Hansschlegelia and Tistrella genera. Research for the genes that encoding for degradation enzymes in isolated genera genome data deposited in Genbank reveled the presence of degradation gene in three species Sinorhizobium meliloti, Novosphingobium panipatenseand Tistrella mobilis.
\end{abstract}

Keywords: crude oil polluted soil, oil refinery sludge, hydrocarbon-degrading bacteria, diversity, 16S rRNA gene sequencing, hydrocarbon-degradation genes

\section{INTRODUCTION}

Petroleum hydrocarbons are the most common environmental pollutants, their release into the environnement, whether accidentally or due to human activities poses a great hazard to terrestrial and marine ecosystems (Holliger et al., 1997). The oil industry including, transport, storage, processing or use generates a significant quantity of sludge during the petroleum-refining process and as the installations are cleaned (Cerqueira et al., 2011). The sludges that result from this treatment process have a high content of petroleum derived hydrocarbons, mainly alkanes and paraffins of 1-40 carbon atoms, along with cycloalkanes and aromatic compounds (Overcash and Pal, 1979). The soil contamination by petroleum hydrocarbons which are known to belong to the family of carcinogens and neurotoxic organic pollutants (Abioye, 2011) causes a significant decline in its quality and such soils become unusable (Gojgic-Cvijovic et al., 2012). Algeria, whose national economy depends on petroleum exploitation, is not an exception to this pollution problem. The Arzew refinery, considered as one of the biggest and most profitable in the North Africa, the third of the country, possesses a high crude oil processing capacity, the various by-products stemming from the refining allow to satisfy fuel, lubricants, asphalt needs of the local population and also to export the surplus products (Naphta and Fuel). However, it is also accompanied by the annual discharge of an important quantity of sludge during the storage tank cleaning operations, which engenders hydrocarbon soil pollution. This is why, the need to clean up crude oil contaminated site is necessary. Bioremediation is an economically and environmentally-friendly procedure based on the metabolic capacities of microorganisms to degrade contaminants biochemically (Huang et al., 2013). Bacteria in particular are considered to be the dominant agents of hydrocarbon degradation in the environment (Röling et al., 2002). Biodegradation using autochthonous bacterial strains is a valuable bioremediation strategy, these indigenous microbes are more likely to survive and proliferate when reintroduced into the site (Silva $\boldsymbol{e t}$ al., 2009; Madueño et al., 2011; Fukuhara et al., 2013; Shankar et al., 2014). The aim of the present work is to isolate autochnonous bacterial strains from polluted soil and oil sludge samples in Arzew refinery. The distribution of genes coding for twenty three enzymes degrading hydrocarbon will be also analyzed from hydrocarbon degrading genera genome data deposited in GenBank and compared to the isolated genera in this study.

\section{MATERIALS AND METHODS}

\section{Site description and sampling}

Oil polluted soil and oil sludge samples were collected from the Arzew refinery $\left(35^{\circ} 49^{\prime} 21.29^{\prime \prime} \mathrm{N} 0^{\circ} 18^{\prime} 37.58^{\prime \prime} \mathrm{O}\right)$ in Northern Algeria (Sonatrach, Algerian Petroleum Company). Two soil samples were taken from the surrounding of the storage tank of crude oil at different period (soil A and soil B on 12.02.2015 and 26.02.2015 respectively). Each sample was a mixture of five sub-samples. A scoop was used to remove organic particles from the soil surface. Soil was collected at 0 to $20 \mathrm{~cm}$ of depth randomly, air dried and sieved $(2 \mathrm{~mm})$ and stored in a dry place at room temperature $\left(\sim 28^{\circ} \mathrm{C}\right)$. Two oil sludge samples were taken from the quagmire of the Arzew refinery, sampling were performed at two different dates (sludge $\mathrm{C}$ and sludge $\mathrm{D}$ on 12.02.2015 and 26.02.2015 respectively) and from 0 to $20 \mathrm{~cm}$ depth. The physicochemical parameters of these soil and sludge samples were analyzed including water content (WC), total nitrogen $\left(\mathrm{N}_{\text {tot }}\right)$ was determined by Kjeldahl digestion, while total phosphorus $\left(\mathrm{P}_{\mathrm{tot}}\right)$ using phosphor molybdic acid, total organic carbon $\left(\mathrm{OC}_{\mathrm{tot}}\right)$ was determined according to Walkley-Black and total petroleum hydrocarbons (TPH) were determined by extracting the samples in methylene chloride, then analyzing the extracts by capillary gas chromatography with flame ionization detection. Oil used in this experiment was provided by the staff of the Arzew refinery and stored in the dark at ambient temperature throughout the study. Before use, oil was sterilized using $0.2 \mu \mathrm{m}$ membrane filter. 


\section{Count and isolation of hydrocarbon-degrading bacteria}

Hydrocarbon-degrading bacteria were isolated from oil sludge and oil contaminated soil samples by using the Bushnell Hass (BH) Mineral Salts (BHMS) medium (Bushnell and Hass, 1941). This medium contains all nutrients except the carbon source. The medium composition is as follows: $\mathrm{MgSO}_{4} \cdot 7 \mathrm{H}_{2} \mathrm{O}$ $(0.2 \mathrm{~g} / \mathrm{L}), \mathrm{K} 2 \mathrm{HPO} 4(1.0 \mathrm{~g} / \mathrm{L}), \mathrm{KH}_{2} \mathrm{PO}_{4}(1.0 \mathrm{~g} / \mathrm{L}), \mathrm{FeCl}_{3}(0.05 \mathrm{~g} / \mathrm{L}), \mathrm{NH}_{4} \mathrm{NO}_{3}(1.0$ $\mathrm{g} / \mathrm{L})$ and $\mathrm{CaCl}_{2}(0.02 \mathrm{~g} / \mathrm{L})$ at $\mathrm{pH} 7.2$, agar $(20 \mathrm{~g} / \mathrm{L})$ was added for $\mathrm{BH}$ agar. The source of carbon and energy was provided either by the addition of sludge in BHMS medium before autoclaving or by adding sterilized oil in the BHMS medium after autoclaving. The hydrocarbon degrading-bacteria cultured from the contaminated soil (A and B) and oil sludge (C and D) samples were quantified by mixing $1 \mathrm{~g}$ of sample with $9 \mathrm{~mL}$ of sterile physiological serum $(9 \% \mathrm{NaCl})$ for $2 \mathrm{~min}$ at high speed. From this soil suspension, successive 1/10 dilutions were made $\left(10^{-3}, 10^{-4}, 10^{-5}\right)$. An aliquot $(0.1 \mathrm{~mL})$ of each diluted soil suspension was spread on BHMS agar plate with the addition of sludge as carbon source and on BHMS agar plate with $100 \mu \mathrm{l}$ of oil. The plates were incubated at $29^{\circ} \mathrm{C}$ for 1 week. Bacterial hydrocarbon-degrading likely grown over incubation period. Colonies were then counted and the number was expressed as UFC / $g$ of sample, bacterial counts were carried out in triplicate. Colonies presenting different morphological appearance were then sub-cultured, purified and conserved for molecular identification. Three mediums were used for strains subculture: Nutrient agar, Tryptic Soy Agar (TSA) medium and diluted 1/10 TSA medium (Biomeriu, France). The isolated strains were then conserved in their appropriated growth medium supplemented with $50 \%$ v/v glycerol solution at $80^{\circ} \mathrm{C}$. For day-to-day experimentation strains were maintained on their growth medium slants at $4{ }^{\circ} \mathrm{C}$ in refrigerator and sub-cultured at an interval of 30 days.

\section{DNA Extraction}

DNA from bacterial cells was extracted using NucleoSpin ${ }^{\circledR} 96$ tissue-vacuum processing (Genomic DNA from tissue and cells, MACHEREY-NAGEL, Hoerdt, France), the strains were cultured for $72 \mathrm{~h}$ at $29^{\circ} \mathrm{C}$ in $150 \mu 1$ of Nutrient Broth (NB) or TSA medium depending on their growth requirements, then $50 \mathrm{ml}$ of bacteria cultures were transferred in $150 \mathrm{ml}$ of medium (Nutrient Broth or TSA) and were incubated until the appearance of a bacterial disorder, the cultures were then centrifuged at $4000 \mathrm{rpm} / \mathrm{min} ; 4{ }^{\circ} \mathrm{C}$ for $30 \mathrm{~min}$, the obtained bacterial pellets were frozen at $-80^{\circ} \mathrm{C}$. Lysis was achieved with the NucleoSpin ${ }^{\circledR} 96$ tissue method by incubation of the bacterial pellets in a solution containing Sodium Dodecyl Sulfate (SDS) and proteinase K. Appropriate conditions for binding of DNA to the silica membrane in the NucleoSpin $® 96$ tissue columns were created by addition of large amounts of chaotropic salt and ethanol to the lysate. The binding process was reversible and specific to nucleic acids. Contaminations were removed by washing with two different buffers. Pure genomic DNA was finally eluted under low ionic strength conditions in a slightly alkaline elution buffer and wasstored at $-20{ }^{\circ} \mathrm{C}$ until $16 \mathrm{~S}$ ribosomal RNA (rRNA) gene amplification.

\section{PCR amplification of bacterial 16S rRNA genes}

Bacterial 16S rRNA genes were amplified from all DNA samples using the bacterial specific primers pA (5'AGA GTT TGA TCC TGG CTC AG3') and pH (5'AAG GAG GTG ATC CAG CCG CA3') (Edwards and al. 1989).
Amplification reaction was performed using Platinum ${ }^{\circledR}$ PCR SuperMix 96 (Invitrogen, France), each plate well contains anti-Taq DNA polymerase antibody, $\mathrm{Mg}++$, deoxyribonucleotide triphosphates (dNTP), and recombinant Taq DNA Polymerase and each $22.5 \mu$ l volume of Platinum ${ }^{\circledR}$ PCR SuperMix well was supplied at $1.1 \mathrm{X}$ concentration to allow approximately $10 \%$ of the final reaction volume to be used for the addition of $1 \mu 1$ of each primer and $2 \mu 1$ of DNA solution (10 to 50ng). The PCR amplification program was conducted as follows: Incubate at $94^{\circ} \mathrm{C}$ for $5 \mathrm{~min}$ to activate the enzyme, and then perform 30 cycles of PCR. Denature $94^{\circ} \mathrm{C}$ for $15 \mathrm{~s}$, Anneal $55^{\circ} \mathrm{C}$ for $30 \mathrm{~s}$, Extend $72^{\circ} \mathrm{C}$ for $1 \mathrm{~min} 30 \mathrm{~s}$ then hold at $10^{\circ} \mathrm{C}$. Amplified DNA was verified in $1 \%$ agarose gel electrophoresis.

\section{Sequencing and phylogenetic analysis}

PCR products were sequenced by GATC Biotech AG (Constance, Allemagne); $20 \mu \mathrm{l}$ of PCR products were sent to sequence. The sequences were corrected by the SeqMan Pro softwwere (http://www.dnastar.com) before alignment and analysis of the 16S rRNA gene sequences using CLUSTAL X software (version 2.0) (http://www.clustal.org). Taxonomic affiliation was done by analyzing similarities using Blast on NCBI (Altschul et al., 1990). A phylogenetic dendrogram was constructed by the Neighbor Joining Method and tree topology as evaluated by performing boot-strap using Mega 6.06.

\section{Distribution of genes encoding for hydrocarbon degrading enzymes}

Twenty-seven proteins sequences listed in Table 4 were selected as reference sequence of hydrocarbon dergradation and investigated for their different encoding genes. The conserved proteomic domains were checked in total sequenced gonome database from GenBank for nine genera (Sinorhizobium, Promicromonospora, Novosphingobium, Georgenia, Starkeya, Roseomonas, Ancylobacter and Hansschlegelia). These specific genes were highlited for genera level.

\section{RESULTS AND DISCUSSION}

\section{Soils and sludges characteristics, counts of culturable hydrocarbon-} degrading bacteria

The physico-chemical analysis of crude oil polluted soil and oil refinery sludge samples (Table 1) revealed the following $\mathrm{C}$ : $\mathrm{N}$ : $\mathrm{P}$ ratio: Polluted soil A (1 :1.2 : $2.5)$, Polluted soil B $(1: 1.25: 3.35)$, Oil refinery sludge $C(3.17: 1: 1.23)$, Oil refinery $\mathrm{D}(1.3: 1: 2.6)$, while for bioremediation operations, US EPA recommended a ratio of 100:10:1 in soil for appropriate nutrients to stimulate microorganism growth (U.S. EPA, 2002). This study reported that biostimulation (i.e., addition of nutrients $\mathrm{N}$ and $\mathrm{P}$ ) strategies are essential to enhance bioremediation of the crude oil contaminated soil and oil refinery sludge collected from Arzew rafinery. Enumeration of culturable bacteria in soil and sludge samples (Table 2) demonstrated that significant hydrocarbon degrading bacteria populations were present in all samples. A number from $0.74 \times 10^{5}$ to $1.8 \times 10^{5} \mathrm{CFU} / \mathrm{g}$ are counted in the sludge medium and from $0.82 \times 10^{5}$ to $2.02 \times 10^{5}$ $\mathrm{CFU} / \mathrm{g}$ in the petroleum medium. The same concentration was found in the soil contaminated with crude petroleum oil in Jordan (Saadoun, 2002).

Table 1 Physicochemical features of soil and sludge samples

\begin{tabular}{|c|c|c|c|c|c|}
\hline Sample & $\begin{array}{l}\text { WC } \\
(\%)\end{array}$ & $\begin{array}{l}\mathrm{N}_{\text {tot }} \\
(\%)\end{array}$ & $\begin{array}{l}\mathbf{P}_{\text {tot }} \\
(\%)\end{array}$ & $\begin{array}{l}\mathbf{O C}_{\text {tot }} \\
(\%)\end{array}$ & $\begin{array}{c}\text { TPH } \\
\left(\mathrm{mg}^{\left.-k^{-1}\right)}\right)\end{array}$ \\
\hline Crude oil polluted soil A & 2.58 & 4.16 & 9.20 & 13.69 & 353 \\
\hline Crude oil polluted soil B & 1.54 & 2.28 & 6.13 & 19.83 & 183 \\
\hline Oil refinery sludge $\mathrm{C}$ & 14.95 & 13.08 & 16.87 & 55.10 & 4153 \\
\hline Oil refinery sludge D & 18.03 & 10.58 & 13.80 & 57.06 & 2776 \\
\hline
\end{tabular}

Table 2 Counts of hydrocarbons-degrading bacteria on oil refinery sludge and petroleum medium

\begin{tabular}{lcc}
\hline Sample & $\begin{array}{c}\text { bacterial count on oil refinery sludge } \\
\text { medium }\end{array}$ & $\begin{array}{c}\text { bacterial count on petroleum } \\
\text { medium }\end{array}$ \\
\hline Crud oil polluted soil A & $0.79 \times 10^{5} \pm 0.17$ & $2.02 \times 10^{5} \pm 0.38$ \\
Crud oil polluted soil B & $1.8 \times 10^{5} \pm 0.51$ & $1.53 \times 10^{5} \pm 0.46$ \\
Oil refinery sludge C & $1.06 \times 10^{5} \pm 0.09$ & $0.91 \times 10^{5} \pm 0.21$ \\
Oil refinery sludge D & $0.74 \times 10^{5} \pm 0.17$ & $0.82 \times 10^{5} \pm 0.21$ \\
\end{tabular}

\section{Identification of hydrocarbon-degrading bacterial isolates}

Fifty-eight bacterial strains were isolated from polluted soil and oil refinery sludge on petroleum and oil refinery sludge medium, cultures were maintained at $29^{\circ} \mathrm{C}$ for 1 week. Based on phenotypic characteristics (colony morphology, pigmentation, growth properties), 22 strains were selected for 16S rRNA gene sequencing. The comparison of obtained sequences by BLAST in Genbank showed that the majority of these strains (20/22) belonged to the phylum Proteobacteria (Table 3), with a unique class of Alphaproteobacteria; the two remaining strains belonged to the phylum Actinobacteria. Many studies on the 
bacterial diversity in a hydrocarbon-contaminated soil, whether by culture-based methods or molecular methods, proved the dominance of Proteobacteria in hydrocarbonscontaminated soils (Saul et al., 2005; Uhlik et al., 2012; Zhang et al., 2012; Mao et al., 2012). The genus Rhizobium (9 strains) was dominating among Alphaproteobacteria; with a singlespecies Sinorhizobium meliloti (showed 100\% 16S rRNA of gene sequence similarity). Other representatives of this class were related to the genera Novosphingobium (4 strains) with two species Novosphingobium naphthalenivorans (99\% of similarity) and Novosphingobium panipatense (99\% of similarity), Tistrella (4 strains), Roseomonas (1), Hansschlegelia (1) and Ancylobacter (1). The Actinobacteria comprised two genera Promicromonospora (1) and Georgenia (1). Zhang et al., (2012) revealed a bacterial diversity close to the identified strains in this study, dominated by the phylum Proteobacteria with a majority of strains belonging to the genus Rhizobium in addition to both genera Roseomonas and Tistrella. The construction of a phylogenetic tree by the neighbor-joining method permitted grouping the isolates into 8 clusters (Figure 1).

The first cluster was close to Sinorhizobium meliloti, including SA1, SA12, SA13, SA14, SA15 and SA17 strains isolated from oil polluted soil A and SB4 SB5 and SB6 strains isolated from oil polluted soil B. Rhizobia survival under hydrocarbon contamination and the interaction of these bacteria with pollutan was reported by several authors (Andreoni et al.,2004; Keum et al., 2006 Poonthrigpun et al., 2006 ; Wang et al., 2007), Sinorhizobium meliloti species had been isolated from hydrocarbon contaminated soil (Ahmadet al., 1997; Muratova, 2014 ; Abou-Shanab, 2016 ) and had shown their ability to degrade various forms of hydrocarbon such as aromatic compounds (Frassinetti et al., 1998) and PCB (Poly Chlororinated Biphenyl) (Wang et al., 2016) Interestingly, the ability for N-fixation of the genus Rhizobium and their presence in the rhizosphere of oil-contaminated soils could play a major role in phytoremediation (Jurelevicius et al., 2010; Xu et al., 2010)thanks to their legumessymbiotic interaction (Johnson et al., 2004; Suominen et al., 2000). The second cluster comprised one strain SB15 isolated from oil polluted soil B and it was close to Hansschlegelia zhihuaiae. The genus Hansschlegelia is a member of family Methylocystaceae (Ivanova et al., 2010) that belonged to the order Rhizobiales, within the class Alphaproteobacteria. This family was classified as type II methanotrophs, which was able to utilize methane and its derivatives as carbon sources through the serine pathway. Many of these methanotrophic bacteria performed important environmental functions as they were a link in the global carbon cycle, acted as nitrogen fixers, and have the ability to degrade a variety of organic contaminants (Gulledge et al., 2001). Hansschlegelia zhihuaiae was isolated from a polluted-soil sample (Wen et al., 2011), and known for its degradation ability of a variety of sulfonylurea herbicides (Hang $\boldsymbol{e}$ al., 2012), whereas there are no reports for their ability to survive in hydrocarbon polluted soils. This is the first time where Hansschlegelia zhihuaiae shows ability to survive in oil polluted soil. The third cluster included one strain SB7 isolated from oil polluted soil B and it was close to Starkeya koreensi (Blast in NCBI), this species was isolated from rice straw (Im $\boldsymbol{e t}$ al., 2006), however another species Starkeya novella was isolated from agricultural soil and employed a mixotrophic growth strategy, Starkeya novella was a facultative sulfur oxidizing chemolithoautotroph, but is also capable of utilizing various carbon compounds, including methanol, for growth as well as atleast 39 reduced carbon sources including sugars, amino sugars, amino acids, and organic acids (Starkey, 1935; Chandra and Shethna, 1977; Kelly et al., 2000; Kappler et al., 2012). Since Starkeya novella used a wide range of carbon compounds for its metabolism, this could explain its presence in soils polluted by hydrocarbons. Phylogenetically, strain SB7 was related with Ancylobacter dichloromethanicus species, this strain was isolated from the polluted soil and was utilized dichloromethane, methanol, formate and formaldehyde along with a variety of polycarbon compounds (Firsova et al., 2009). The fourth cluster grouped four strains ORSA6, ORSA7, SA9 and ORSA5 isolated from oil contaminated soil A and oil refinery sludge A and were close to two species Novosphingobium naphthalenivorans and Novosphingobium panipatense, respectively. Several aromatic-degrading strains of Novosphingobium such as Novosphingobium aromaticivorans, Novosphingobium naphthalenivorans, Novosphingobium pentaromaticivorans, Novosphingobium panipatense and Novosphingobium naphthaehad been reported (Kertesz and Kawasaki, 2010; Gupta, 2009; Suzuki and Hiraishi, 2007; Lyu, 2014; Chaudhary and Kim, 2016).

Table 3 Origin, isolation medium and genotypic characteristics of isolated strains

\begin{tabular}{|c|c|c|c|c|c|}
\hline Strain & Origin & Isolation medium & Acc. Num. & Nearest phylogenetic neighbor (\% Similarity) & Phylum \\
\hline SA1 & Pollued soil A & petroleum & MG786631 & Sinorhizobium meliloti strain WSM1022 100\% & Alphaproteobacteria \\
\hline SA3 & Pollued soil A & petroleum & MG786632 & Promicromonospora aerolata strain $1262399 \%$ & Actinobacteria \\
\hline SA9 & Pollued soil A & petroleum & MG786633 & $\begin{array}{l}\text { Novosphingobium naphthalenivorans strain VIT- } \\
\text { DD2 } 99 \%\end{array}$ & Alphaproteobacteria \\
\hline SA12 & Pollued soil A & petroleum & MG786634 & Sinorhizobium meliloti strain LMTR32 100\% & Alphaproteobacteria \\
\hline SA13 & Pollued soil A & petroleum & MG786635 & Sinorhizobium meliloti strain LLAN18 100\% & Alphaproteobacteria \\
\hline SA14 & Pollued soil A & petroleum & MG786636 & Sinorhizobium meliloti strain LMTR32 100\% & Alphaproteobacteria \\
\hline SA15 & Pollued soil A & oil refinery sludge & MG786637 & Sinorhizobium meliloti strain LLAN18 100\% & Alphaproteobacteria \\
\hline SA16 & Pollued soil A & oil refinery sludge & MG786638 & Georgenia ferrireducens strain F64 99\% & Actinobacteria \\
\hline SA17 & Pollued soil A & oil refinery sludge & MG786639 & Sinorhizobium meliloti strain LLAN18 100\% & Alphaproteobacteria \\
\hline SB4 & Pollued soil B & oil refinery sludge & MG786640 & Sinorhizobium meliloti strain LLAN18 100\% & Alphaproteobacteria \\
\hline SB5 & Pollued soil B & oil refinery sludge & MG786641 & Sinorhizobium meliloti strain LLAN18 100\% & Alphaproteobacteria \\
\hline SB6 & Pollued soil B & oil refinery sludge & MG786642 & Sinorhizobium meliloti strain LLAN18 100\% & Alphaproteobacteria \\
\hline SB7 & Pollued soil B & oil refinery sludge & MG786643 & Starkeya koreensis strain NBRC 100963 97\% & Alphaproteobacteria \\
\hline SB11 & Pollued soil B & petroleum & MG786644 & Roseomonas ludipueritiae $97 \%$ & Alphaproteobacteria \\
\hline SB15 & Pollued soil B & petroleum & MG786645 & Hansschlegelia zhihuaiae strain S $11399 \%$ & Alphaproteobacteria \\
\hline ORSA5 & oil refinery sludge $\mathrm{C}$ & oil refinery sludge & MG786624 & Novosphingobium panipatense strain SM16 99\% & Alphaproteobacteria \\
\hline ORSA7 & oil refinery sludge $\mathrm{C}$ & oil refinery sludge & MG786625 & $\begin{array}{c}\text { Novosphingobium naphthalenivorans strain VIT- } \\
\text { DD2 } 99 \%\end{array}$ & Alphaproteobacteria \\
\hline ORSA6 & oil refinery sludge $\mathrm{C}$ & petroleum & MG786626 & $\begin{array}{l}\text { Novosphingobium naphthalenivorans strain VIT- } \\
\text { DD2 } 99 \%\end{array}$ & Alphaproteobacteria \\
\hline ORSB2 & oil refinery sludge $\mathrm{D}$ & oil refinery sludge & MG786627 & Tistrella mobilis strain DSD-PW4-OH23 100\% & Alphaproteobacteria \\
\hline ORSB4 & oil refinery sludge D & oil refinery sludge & MG786628 & Tistrella mobilis strain DSD-PW4-OH23 100\% & Alphaproteobacteria \\
\hline ORSB5 & oil refinery sludge D & petroleum & MG786629 & Tistrella mobilis strain DSD-PW4-OH23 100\% & Alphaproteobacteria \\
\hline ORSB6 & oil refinery sludge D & petroleum & MG786630 & Tistrella mobilis strain DSD-PW4-OH23 100\% & Alphaproteobacteria \\
\hline
\end{tabular}


Table 4 Distribution of genes coding for degradation enzymes in isolated genera genome data deposited in Genbank

\begin{tabular}{|c|c|c|c|c|c|c|c|c|c|c|}
\hline Degadation enzymes & Genes & $\begin{array}{l}\text { Sinorhizobium } \\
\text { genera }\end{array}$ & $\begin{array}{c}\text { Promicromonospora } \\
\text { genera }\end{array}$ & $\begin{array}{l}\text { Novosphingobium } \\
\text { genera }\end{array}$ & $\begin{array}{l}\text { Georgenia } \\
\text { genera }\end{array}$ & $\begin{array}{c}\text { Starkeya } \\
\text { genera }\end{array}$ & $\begin{array}{l}\text { Ancylobacter } \\
\text { genera }\end{array}$ & $\begin{array}{l}\text { Roseomonas } \\
\text { genera }\end{array}$ & $\begin{array}{l}\text { Methylocystaceae } \\
\text { family }\end{array}$ & $\begin{array}{l}\text { Tistrella } \\
\text { genera }\end{array}$ \\
\hline $\begin{array}{l}\text { benzoate dioxygenase subunit } \\
\text { alpha }\end{array}$ & benA & S.americanum & & $\begin{array}{l}\text { N.aromaticivorans } \\
\text { N.naphthalenivorans }\end{array}$ & 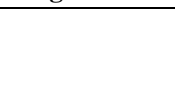 & & & $\begin{array}{l}\text { R.stagni } \\
\text { R.gilardii }\end{array}$ & & \\
\hline $\begin{array}{l}\text { benzoate/naphthalene } \\
\text { dioxygenase large alpha subunit }\end{array}$ & XylX/nahAc & & & $\begin{array}{l}\text { N.aromaticivorans } \\
\text { N.panipatense }\end{array}$ & & & & R.aerilata & Methylosinus $s p$ & \\
\hline $\begin{array}{l}\text { Benzene/toluene/chlorobenzene/e } \\
\text { thylbenzene/naphtalene/cumen } \\
\text { dioxygenase subunit alpha }\end{array}$ & $\begin{array}{l}\text { nagAc/pahAc/p } \\
\text { hnAc/etbA1/nar } \\
\text { Aa/nidA/pdoA2 } \\
\text { /cumA1/todC1/ } \\
\text { bphA/bphA1/ph } \\
\text { dA }\end{array}$ & S.saheli & & $\begin{array}{l}\text { N.aromaticivorans } \\
\text { N.naphthalenivorans } \\
\text { N.panipatense }\end{array}$ & & & & & & T.mobilis \\
\hline $\begin{array}{l}\text { salicylate-5-hydroxylase large } \\
\text { oxygenase component }\end{array}$ & nagG & & & $\begin{array}{l}\text { N.aromaticivorans } \\
\text { N.naphthalenivorans } \\
\text { N.panipatense }\end{array}$ & & & & & & T.mobilis \\
\hline $\begin{array}{l}\text { benzoate/cumen/ethylbenzene } \\
\text { dioxygenase small beta subunit }\end{array}$ & $\begin{array}{l}\text { benB/XylY/pdo } \\
\text { B2/phnAd/phdB } \\
\text { /nidB/bphA2/cu } \\
\text { mA2 /etbA2 }\end{array}$ & $\begin{array}{l}\text { S.americanum } \\
\text { S.saheli }\end{array}$ & & $\begin{array}{l}\text { N.aromaticivorans } \\
\text { N.naphthalenivorans } \\
\text { N.panipatense }\end{array}$ & & & & $\begin{array}{l}\text { R.stagni } \\
\text { R.gilardii } \\
\text { R.aerilata }\end{array}$ & & T.mobilis \\
\hline $\begin{array}{l}\text { naphthalene dioxygenase small } \\
\text { beta subunit }\end{array}$ & $\begin{array}{l}\text { nahAd/nagAd/p } \\
\text { ahAd }\end{array}$ & & & $\begin{array}{l}\text { N.aromaticivorans } \\
\text { N.naphthalenivorans }\end{array}$ & & & & & & \\
\hline $\begin{array}{l}\text { salicylate-5-hydroxylase small } \\
\text { oxygenase component }\end{array}$ & nagH & & & $\begin{array}{l}\text { N.aromaticivorans } \\
\text { N.naphthalenivorans } \\
\text { N.panipatense }\end{array}$ & & & & $\begin{array}{l}\text { R.stagni } \\
\text { R.gilardii }\end{array}$ & & T.mobilis \\
\hline dioxygenase small alpha subunit & nidB (Rhodo) & & 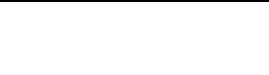 & $\begin{array}{l}\text { N.aromaticivorans } \\
\text { N.naphthalenivorans }\end{array}$ & & & & & & \\
\hline catechol 1,2-dioxygenase & catA & S.americanum & P. kroppenstedtii & $\begin{array}{l}\text { N.aromaticivorans } \\
\text { N.naphthalenivorans } \\
\text { N.panipatense }\end{array}$ & & & & $\begin{array}{l}\text { R.stagni } \\
\text { R.gilardii }\end{array}$ & & \\
\hline $\begin{array}{l}\text { biphenyl-2,3-diol 1,2- } \\
\text { dioxygenase /1,2- } \\
\text { dihydroxynaphthalene } \\
\text { dioxygenase }\end{array}$ & $\begin{array}{l}\text { bphC } \\
\text { /nahC/doxG/etb } \\
\text { C/pahC }\end{array}$ & & & $\begin{array}{l}\text { N.aromaticivorans } \\
\text { N.naphthalenivorans } \\
\text { N.panipatense }\end{array}$ & & & & R.aerilata & & \\
\hline catechol-2,3-dioxygenase & nahH & & & $\begin{array}{l}\text { N.aromaticivorans } \\
\text { N.naphthalenivorans } \\
\text { N.panipatense }\end{array}$ & & & & $\begin{array}{l}\text { R.stagni } \\
\text { R.gilardii } \\
\text { R.aerilata }\end{array}$ & & \\
\hline $\begin{array}{l}1 \text { 4-hydroxybenzoate 3- } \\
\text { monooxygenase }\end{array}$ & HBM & $\begin{array}{l}\text { S.americanum } \\
\text { S.saheli } \\
\text { S.meliloti }\end{array}$ & P. kroppenstedtii & $\begin{array}{l}\text { N.aromaticivorans } \\
\text { N.panipatense }\end{array}$ & Georgenia sp. & & & $\begin{array}{l}\text { R.stagni } \\
\text { R.gilardii } \\
\text { R.aerilata }\end{array}$ & & T.mobilis \\
\hline alkane-1 monooxygenase & alkB & & & $\begin{array}{l}\text { N.aromaticivorans } \\
\text { N.panipatense } \\
\end{array}$ & & & & & & \\
\hline salicylate hydroxylase & nahG/nahW & $\begin{array}{l}\text { S.americanum } \\
\text { S.saheli }\end{array}$ & P. kroppenstedtii & $\begin{array}{l}\text { N.aromaticivorans } \\
\text { N.naphthalenivorans }\end{array}$ & Georgenia sp. & S.novella & $\begin{array}{l}\text { Ancylobacter sp. } \\
\text { A.rudongensis }\end{array}$ & $\begin{array}{l}\text { R.stagni } \\
\text { R.gilardii }\end{array}$ & $\begin{array}{l}\text { M.trichosporium } \\
\text { Methylopila sp. }\end{array}$ & T.mobilis \\
\hline
\end{tabular}


J Microbiol Biotech Food Sci / Sekkour et al. 2019 : 9 (1) 70-77

\begin{tabular}{|c|c|c|c|c|c|c|c|c|c|c|}
\hline & & S.meliloti & & N.panipatense & & & & R.aerilata & & \\
\hline $\begin{array}{l}1 \text { 2-carboxybenzaldehyde } \\
\text { dehydrogenase }\end{array}$ & phdK & S.meliloti & & N.naphthalenivorans & & & & R.aerilata & Methylopila $s p$ & \\
\hline $\begin{array}{l}\text { naphthalene } \\
\text { dioxygenase/ferredoxin }\end{array}$ & $\begin{array}{l}\text { doxA/NahAb/na } \\
\text { gAb/pahAb }\end{array}$ & $\begin{array}{l}\text { S.americanum } \\
\text { S.saheli } \\
\text { S.meliloti }\end{array}$ & P. kroppenstedtii & $\begin{array}{l}\text { N.aromaticivorans } \\
\text { N.naphthalenivorans } \\
\text { N.panipatense }\end{array}$ & Georgenia sp. & & & $\begin{array}{l}\text { R.stagni } \\
\text { R.gilardii } \\
\text { R.aerilata }\end{array}$ & & T.mobilis \\
\hline $\begin{array}{l}\text { reductase naphthalene } \\
\text { dioxygenase/ ferredoxin } \\
\text { reductase }\end{array}$ & $\begin{array}{l}\text { nahAa/nagAa/p } \\
\text { ahAa }\end{array}$ & S.saheli & & $\begin{array}{l}\text { N.aromaticivorans } \\
\text { N.naphthalenivorans } \\
\text { N.panipatense }\end{array}$ & & & & & & T.mobilis \\
\hline $\begin{array}{l}\text { benzoate 1,2-dioxygenase } \\
\text { ferredoxin }\end{array}$ & benC & S.americanum & & & & & & & & \\
\hline salicylaldehyde dehydrogenase & $\begin{array}{l}\text { PhnF/nahF/dox } \\
\text { F/nagF/pahF }\end{array}$ & S.saheli & P. kroppenstedtii & $\begin{array}{l}\text { N.aromaticivorans } \\
\text { N.naphthalenivorans } \\
\text { N.panipatense }\end{array}$ & & & & $\begin{array}{l}\text { R.gilardii } \\
\text { R.aerilata }\end{array}$ & & \\
\hline $\begin{array}{l}\text { 2-hydroxymuconic semialdehyde } \\
\text { dehydrogenase }\end{array}$ & nahI & & & $\begin{array}{l}\text { N.aromaticivorans } \\
\text { N.naphthalenivorans } \\
\text { N.panipatense }\end{array}$ & & & & & & T.mobilis \\
\hline $\begin{array}{l}\text { Cis-naphthalene dihydrodiol } \\
\text { dehydrogenase }\end{array}$ & $\begin{array}{l}\text { nahB/doxE/nag } \\
\text { B/phnB/pahB/ni } \\
\text { dC/bphB }\end{array}$ & S.saheli & & $\begin{array}{l}\text { N.aromaticivorans } \\
\text { N.naphthalenivorans } \\
\text { N.panipatense }\end{array}$ & & & & & & T.mobilis \\
\hline hydratase-aldolase & $\begin{array}{l}\text { nahE/phnE/pah } \\
\text { E/nidD }\end{array}$ & & & $\begin{array}{l}\text { N.aromaticivorans } \\
\text { N.naphthalenivorans } \\
\text { N.panipatense }\end{array}$ & & & & & & \\
\hline $\begin{array}{l}\text { 2-hydroxychromene-2- } \\
\text { carboxylate isomerase }\end{array}$ & $\begin{array}{l}\text { nahD/phnD/pah } \\
\mathrm{D}\end{array}$ & S.americanum & & $\begin{array}{l}\text { N.aromaticivorans } \\
\text { N.naphthalenivorans } \\
\text { N.panipatense }\end{array}$ & & S.novella & $\begin{array}{l}\text { Ancylobacter sp. } \\
\text { A.rudongensis }\end{array}$ & $\begin{array}{l}\text { R.stagni } \\
\text { R.aerilata }\end{array}$ & Methylopila sp & T.mobilis \\
\hline $\begin{array}{l}\text { 2-hydroxymuconic semialdehyde } \\
\text { hydrolase }\end{array}$ & nahN & S.saheli & & $\begin{array}{l}\text { N.aromaticivorans } \\
\text { N.naphthalenivorans } \\
\text { N.panipatense }\end{array}$ & & & & & & T.mobilis \\
\hline Ferredoxin & phdC & & P. kroppenstedtii & & & & & & & \\
\hline Ferredoxin reductase & phdD & $\begin{array}{l}\text { S.americanum } \\
\text { S.saheli } \\
\text { S.meliloti } \\
\end{array}$ & P. kroppenstedtii & $\begin{array}{l}\text { N.aromaticivorans } \\
\text { N.naphthalenivorans } \\
\text { N.panipatense }\end{array}$ & Georgenia sp. & S.novella & $\begin{array}{l}\text { Ancylobacter sp. } \\
\text { A.rudongensis }\end{array}$ & $\begin{array}{l}\text { R.stagni } \\
\text { R.aerilata }\end{array}$ & $\begin{array}{l}\text { M.trichosporium } \\
\text { Methylopila sp. }\end{array}$ & T.mobilis \\
\hline extradiol dioxygenase & phnC & S.saheli & & & & & & & & T.mobilis \\
\hline
\end{tabular}




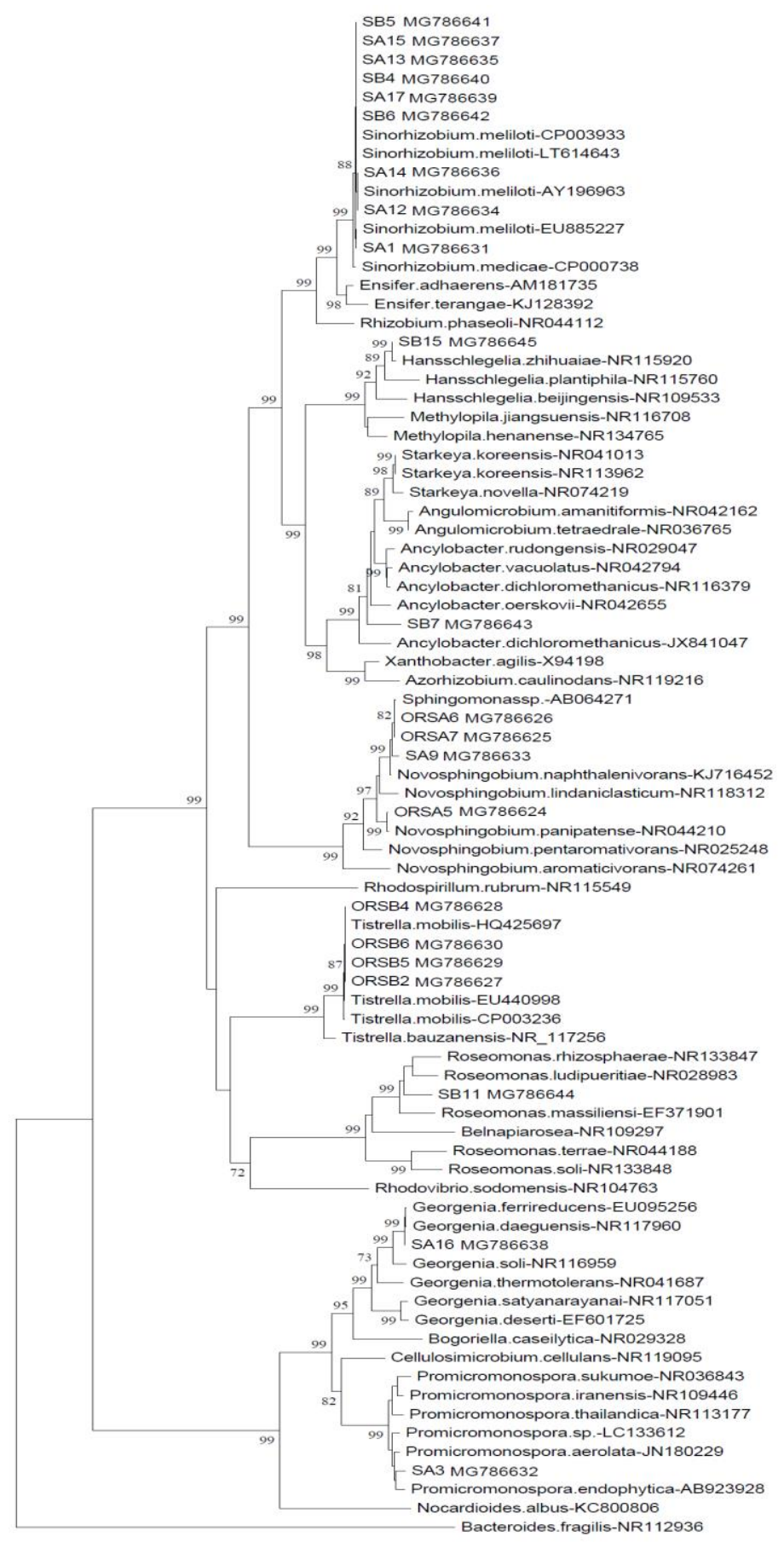

0.05

Figure 1 Phylogenetic tree based on 16SrRNA gene sequence (>900nt) analysis of new and reference strains. Neighbor-joinin method integrating Kimura2 distance was used. Data are bootstrap values issued from 1000 repetitions

The fifth cluster was close to Tistrella mobilis and included four strains, ORSB4, ORSB6, ORSB5 and ORSB2 isolated from oil refinery sludge B. The genus Tistrella was isolated from soil samples contaminated with polycyclic aromatic hydrocarbon (PAH)-containing waste from oil refinery field in Shanghai, China and can increase the speed of phenanthrene degradation (Zhao et al., 2008), the species Tistrella bauzanensis was isolated from soil containing high levels of heavy oil (Zhang et al., 2011)howeverthe species Tistrella mobilis was isolated only from wastewater, sea water and surface sediment collected from deep sea contaminated by hydrocarbons and proved its efficiency in this pollutants degradation (Shi et al., 2002 ; Cuiand Shao, 2009). The sixth cluster comprised one strain SB11 isolated from oil polluted soil B and it was close to Roseomonas ludipueritiae. The genus Roseomonas of the familly Acetobacteraceae belonging to phylum Proteobacteria, was first described by Rihs et al. (1993) associated with bacteraemia and other humain infections. Recently, a new strain Roseomonas nepalensiswas isolated from oil-contaminated soil of Nepal, during a study of oil-utilizing bacteria (Chaudhary and Kim, 2017). The seventh cluster included one strain SA16 isolated from oil polluted soil A and it was close to Georgenia ferrireducens.Franzetti et al.(2011) isolated Georgenia ferrireducens as bacteria able to produce biological emulsifiers from a polycyclic aromatic hydrocarbons (PAHs) contaminated soil. The eighth and last cluster comprised one strain SA3 isolated from oil polluted soil A and it was close to Promicromonospora aerolata. it was reported that Promicromonospora aerolatas pecies was isolated from air (Busse et al., 2003). However, several species belonging to the genus Promicromonospora were isolated from soil; recently, two genera isolated from soil were added: Promicromonospora soli (Zhang $\boldsymbol{e}$ al., 2017) and Promicromonospora kermanensis (Mohammadipanah et al., 2017), however, there was no reports in their ability to survive in hydrocarbon polluted soils.

\section{Distribution of genes coding for dgradation enzymes in isolated genera}

Different genes encoding for hydrocarbons degradation were investigated through Genbank (tBLASTn) databases. Many studies dealing with total genome sequencing revealed the presence of these genes of interest (Table 4), but without proving their real ability to degrade hydrocarbons in vitro (Sinorhizobium americanum, S. saheli, Promicromonospora kroppenstedtii, Starkeya novella, Ancylobacter sp., Methylosinus trichosporium, , Methylopila sp., Ancylobacter rudongensis, Roseomonas stagnii, $R$. gilardi, and $R$. aerilata); Only seven species did degrade hydrocarbons and possessed these genes of interest: Sinorhizobium meliloti which was reported as PAHdegrading (Ahmad et al., 1997) and harbored several putative genes of degradation (Galibert et al., 2001); Novosphingobium aromaticivorans (Fredrickson et al., 1991 , 1995; Romine et al., 1999) ; N. naphthalenivorans (Suzuki and Hiraishi, 2007); N. panipatense (Gupta et al., 2009); Methylosinus sp. (Bowman et al., 1993); Georgenia sp. (Al-Awadhi et al.,2007); Tistrella mobilis (Cui et al., 2009). Finally; Hansschlegelia zhihuaiaedid degrade hydrocarbon in this study but no work did reveal the gens of degradation.

\section{CONCLUSION}

In conclusion a high diversity of hydrocarbon-degrading bacteria were found in crude oil polluted soil and oil refinery sludge from Arzew refinery, culturedmethod allowed to obtain different species known for their degradative capability and to demonstrate species never known for hydrocarbon degradation. A limited number of genes coding for degradation enzymes were revealed in these genera Promicromonospora, Georgenia, Starkeya, Ancylobacter and Methylocystaceae family. However, as a perspective it is interesting to look for these genes of interest in the isolated species belonging in these genera or complete sequencing of the genome.

\section{REFERENCES}

ABIOYE, O. P. 2011. Biological remediation of hydrocarbon and heavy metals contaminated soil. In Soil Contamination. InTech. https://doi.org/10.5772/24938 ABOU-SHANAB, R. A. I., ERAKY, M., HADDAD, A. M., ABDEL-GAFFAR, A. B., SALEM, A. M. 2016. Characterization of Crude Oil Degrading Bacteria Isolated from Contaminated Soils Surrounding Gas Stations. Bulletin of environmental contamination and toxicology, 97(5), 684-688. https://dx.doi.org/10.1007/s00128-016-1924-2

AHMAD, D., MEHMANNAVAZ, R., DAMAJ, M. 1997. Isolation and characterization of symbiotic N2-fixing Rhizobium meliloti from soils contaminated with aromatic and chloroaromatic hydrocarbons: PAHs and PCBs. International biodeterioration \& biodegradation,39(1), 33-43. https://dx.doi.org/10.1016/S0964-8305(96)00065-0

AL-AWADHI, H., SULAIMAN, R. H., MAHMOUD, H. M., RADWAN, S. S 2007. Alkaliphilic and halophilic hydrocarbon-utilizing bacteria from Kuwaiti coasts of the Arabian Gulf. Applied microbiology and biotechnology, 77(1), 183 186. https://dx.doi.org/10.1007/s00253-007-1127-1

ALTSCHUL, S. F., GISH, W., MILLER, W., MYERS, E. W., LIPMAN, D. J. 1990. Basic local alignment search tool. Journal of molecular biology, 215(3), 403-410. https://dx.doi.org/10.1016/S0022-2836(05)80360-2

ANDREONI, V., CAVALCA, L., RAO, M. A., NOCERINO, G. BERNASCONI, S., DELL'AMICO, E., ... GIANFREDA, L. 2004. Bacterial communities and enzyme activities of PAHs polluted soils. Chemosphere, 57(5), 401-412. https://dx.doi.org/10.1016/j.chemosphere.2004.06.013

BOWMAN, J. P., SLY, L. I., NICHOLS, P. D., HAYWARD, A. C. 1993. Revised taxonomy of the methanotrophs: description of Methylobacter gen. nov., emendation of Methylococcus, validation of Methylosinus and Methylocystis species, and a proposal that the family Methylococcaceae includes only the group I methanotrophs. International Journal of Systematic and Evolutionary Microbiology, 43(4), 735-753. https://dx.doi.org/10.1099/00207713-43-4-735 BUSHNELL, L. D., HAAS, H. F. 1941. The utilization of certain hydrocarbons by microorganisms. Journal of Bacteriology, 41(5), 653

BUSSE, H. J., ZLAMALA, C., BUCZOLITS, S., LUBITZ, W., KÄMPFER, P., TAKEUCHI, M. 2003. Promicromonospora vindobonensis sp. nov. and Promicromonospora aerolata sp. nov., isolated from the air in the medieval 
'Virgilkapelle'in Vienna. International journal of systematic and evolutionary microbiology, 53(5), 1503-1507. https://dx.doi.org/10.1099/ijs.0.02522-0

CERQUEIRA, V. S., HOLLENBACH, E. B., MABONI, F., VAINSTEIN, M H., CAMARGO, F. A., MARIA DO CARMO, R. P., BENTO, F. M. 2011 Biodegradation potential of oily sludge by pure and mixed bacterial cultures. Bioresource technology, 102(23),

https://dx.doi.org/10.1016/j.biortech.2011.09.074

CHANDRA T. S., SHETHNA Y. I. 1977. Oxalate, formate, formamide, and methanol metabolism in Thiobacillus novellus. J. Bacteriol. 131, 389-398 CHAUDHARY, D. K., KIM, J. 2016. Novosphingobium naphthae sp. nov., from oil-contaminated soil. International journal of systematic and evolutionary microbiology, 66(8), 3170-3176. https://dx.doi.org/10.1099/ijsem.0.001164

CHAUDHARY, D. K., KIM, J. 2017. Roseomonas nepalensis sp. nov., isolated from oil-contaminated soil. International journal of systematic and evolutionary microbiology, 67(4), 981-987. https://dx.doi.org/10.1099/ijsem.0.001727

CUI, Z., SHAO, Z. 2009. Predominant strains of polycyclic aromatic hydrocarbon-degrading consortia from deep sea of the Middle Atlantic Ridge. Wei sheng wu xue bao= Acta microbiologica Sinica, 49(7), 902-909. FIRSOVA, J., DORONINA, N., LANG, E., SPRÖER, C., VUILLEUMIER, S., TROTSENKO, Y. 2009. Ancylobacter dichloromethanicus sp. nov. a new aerobic facultatively methylotrophic bacterium utilizing dichloromethane. Systematic and applied microbiology,32(4), 227-232. https://dx.doi.org/10.1016/j.syapm.2009.02.002

FRANZETTI, A., GANDOLFI, I., BERTOLINI, V., RAIMONDI, C. PISCITELlO, M., PAPACCHINI, M., BESTETTI, G. 2011. Phylogenetic characterization of bioemulsifier-producing bacteria. International biodeterioration \& biodegradation, 65(7), 1095-1099. https://dx.doi.org/10.1016/j.jibiod.2011.01.014

FRASSINETTI, S., SETTI, L., CORTI, A., FARRINELLI, P., MONTEVECCHI, P., VALLINI, G. 1998. Biodegradation of dibenzothiophene by a nodulating isolate of Rhizobium meliloti. Canadian journal of microbiology, 44(3), 289-297. https://dx.doi.org/10.1139/w97-155

FREDRICKSON, J. K., BALKWILL, D. L., DRAKE, G. R., ROMINE, M. F., RINGELBERG, D. B., WHITE, D. C. 1995. Aromatic-degrading Sphingomonas isolates from the deep subsurface. Applied and environmental microbiology, 61(5), 1917-1922

FREDRICKSON, J. K., BROCKMAN, F. J., WORKMAN, D. J., LI, S. W., STEVENS, T. O. 1991. Isolation and characterization of a subsurface bacterium capable of growth on toluene, naphthalene, and other aromatic compounds. Applied and environmental microbiology, 57(3), 796-803.

FUKUHARA, Y., HORII, S., MATSUNO, T., MATSUMIYA, Y., MUKAI, M., KUBO, M. 2013. Distribution of hydrocarbon-degrading bacteria in the soil environment and their contribution to bioremediation. Applied biochemistry and biotechnology, 170(2), 329-339. https://dx.doi.org/10.1007/s12010-013-0170-x GALIBERT, F., FINAN, T. M., LONG, S. R., PÜHLER, A., ABOLA, P. AMPE, F., ... BOTHE, G. 2001. The composite genome of the legume symbiont Sinorhizobium meliloti. Science, 293(5530),

$668-672$

https://dx.doi.org/10.1126/science.1060966

GOJGIC-CVIJOVIC, G. D., MILIC, J. S., SOLEVIC, T. M., BESKOSKI, V. P., ILIC, M. V., DJOKIC, L. S., ... VRVIC, M. M. 2012. Biodegradation of petroleum sludge and petroleum polluted soil by a bacterial consortium: a laboratory study. Biodegradation, 23(1),

$1-14$ https://dx.doi.org/10.1007/s10532-011-9481-1

GULLEDGE, J., AHMAD, A., STEUDLER, P. A., POMERANTZ, W. J., CAVANAUGH, C. M. 2001. Family-and genus-level 16S rRNA-targeted oligonucleotide probes for ecological studies of methanotrophic bacteria. Applied and environmental microbiology, 67(10),

4726-4733.

http://dx.doi.org/10.1128/AEM.67.10.4726-4733.2001

GUPTA, S. K., LAL, D., LAL, R. 2009. Novosphingobium panipatense sp. nov. and Novosphingobium mathurense sp. nov., from oil-contaminated soil. International journal of systematic and evolutionary microbiology, 59(1), 156-161. https://dx.doi.org/10.1099/ijs.0.65743-0

HANG, B. J., HONG, Q., XIE, X. T., HUANG, X., WANG, C. H., HE, J., LI, S. P. 2012. SulE, a sulfonylurea herbicide de-esterification esterase from Hansschlegelia zhihuaiae S113. Applied and environmental microbiology, 78(6), 1962-1968. https://dx.doi.org/10.1128/AEM.07440-11

HOLLIGER, C., GASPARD, S., GLOD, G., HEIJMAN, C., SCHUMACHER, W., SCHWARZENBACH, R. P., VAZQUEZ, F. 1997. Contaminated environments in the subsurface and bioremediation: organic contaminants. FEMS Microbiology Reviews, 20(3-4), 517-523. https://dx.doi.org/10.1111/j.15746976.1997.tb00334.x

HUANG, Y., ZENG, Y., YU, Z., ZHANG, J., FENG, H., LIN, X. 2013. In silico and experimental methods revealed highly diverse bacteria with quorum sensing and aromatics biodegradation systems-a potential broad application on bioremediation. Bioresource technology, 148

$311-316$ https://dx.doi.org/10.1016/j.biortech.2013.08.155

IM, W. T., ASLAM, Z., LEE, M., TEN, L. N., YANG, D. C., LEE, S. T. 2006. Starkeya koreensis sp. nov., isolated from rice straw. International journal of systematic and evolutionary microbiology, 56(10), 2409-2414. https://dx.doi.org/10.1099/ijs.0.64093-0
IVANOVA, E., DORONINA, N., TROTSENKO, Y. 2010. Hansschlegelia plantiphila gen. nov., sp. nov. List of New Names and New Combinations Previously Effectively, but not Validly, Published. Validation List, (133), 10091010. https://dx.doi.org/10.1099/ijs.0.024562-0

JOHNSON, D. L., MAGUIRE, K. L., ANDERSON, D. R., MCGRATH, S. P. 2004. Enhanced dissipation of chrysene in planted soil: the impact of a rhizobial inoculum. Soil biology and biochemistry, 36(1), 33-38. https://dx.doi.org/10.1016/j.soilbio.2003.07.004

JURELEVICIUS, D., KORENBLUM, E., CASELlA, R., VITAL, R. L., SELDIN, L. 2010. Polyphasic analysis of the bacterial community in the rhizosphere and roots of Cyperus rotundus L. grown in a petroleum-contaminated soil. J Microbiol Biotechnol, 20(5), $\quad 862-870$. https://dx.doi.org/10.4014/jmb.0910.10012

KAPPLER, U., DAVENPORT, K., BEATSON, S., LUCAS, S., LAPIDUS, A., COPELAND, A., ... TICE, H. 2012. Complete genome sequence of the facultatively chemolithoautotrophic and methylotrophic alpha proteobacterium Starkeya novella type strain (ATCC 8093 T). Standards in genomic sciences, 7(1), 44. https://dx.doi.org/10.4056/sigs.3006378

KELLY, D. P., MCDONALD, I. R., WOOD, A. P. 2000. Proposal for the reclassification of Thiobacillus novellus as Starkeya novella gen. nov., comb. nov., in the alpha-subclass of the Proteobacteria. International journal of systematic and evolutionary microbiology,50(5), 1797-1802. https://dx.doi.org/10.1099/00207713-50-5-1797

KERTESZ, M. A., KAWASAKI, A. 2010. Hydrocarbon-degrading sphingomonads: Sphingomonas, sphingobium, novosphingobium, and sphingopyxis. In Handbook of hydrocarbon and lipid microbiology (pp. 16931705). Springer Berlin Heidelberg. https://dx.doi.org/10.1007/978-3-540-775874_119

KEUM, Y. S., SEO, J. S., HU, Y., LI, Q. X. 2006. Degradation pathways of phenanthrene by Sinorhizobium sp. C4. Applied microbiology and biotechnology, 71 (6), 935-941. https://dx.doi.org/10.1007/s00253-005-0219-z LYU, Y., ZHENG, W., ZHENG, T., TIAN, Y. 2014. Biodegradation of polycyclic aromatic hydrocarbons by Novosphingobium pentaromativorans US61. PloS one, 9(7), e101438. https://dx.doi.org/10.1371/journal.pone.0101438 MADUEÑO, L., COPPOTELLI, B. M., ALVAREZ, H. M., MORELLI, I. S. 2011. Isolation and characterization of indigenous soil bacteria for bioaugmentation of PAH contaminated soil of semiarid Patagonia, Argentina. International biodeterioration \& biodegradation, 65(2), 345-351. https://dx.doi.org/10.1016/j.ibiod.2010.12.008

MAO, J., LUO, Y., TENG, Y., LI, Z. 2012. Bioremediation of polycyclic aromatic hydrocarbon-contaminated soil by a bacterial consortium and associated microbial community changes. International Biodeterioration \& Biodegradation, 70, 141-147. https://dx.doi.org/10.1016/j.j.biod.2012.03.002 MOHAMMADIPANAH, F., DEL CARMEN MONTERO-CALASANZ, M. SCHUMANN, P., SPRÖER, C., ROHDE, M., KLENK, H. P. 2017. Promicromonospora kermanensis sp. nov., an actinobacterium isolated from soil. International journal of systematic and evolutionary microbiology, 67(2), 262-267. https://dx.doi.org/10.1099/ijsem.0.001613

MURATOVA, A., POZDNYAKOVA, N., MAKAROV, O., BABOSHIN, M., BASKUNOV, B., MYASOEDOVA, N., ... TURKOVSKAYA, O. 2014 Degradation of phenanthrene by the rhizobacterium Ensifer meliloti. Biodegradation, 25(6), 787-795. https://dx.doi.org/10.1007/s10532014-9699-9

OVERCASH, M. R., PAL, D. 1979. Design of land treatment systems for industrial wastes-theory and practice. Ann Arbor Science Publishers Inc.

POONTHRIGPUN, S., PATTARAGULWANIT, K., PAENGTHAI, S. KRIANGKRIPIPAT, T., JUNTONGJIN, K., THANIYAVARN, S., PINPHANICHAKARN, P. 2006. Novel intermediates of acenaphthylene degradation by Rhizobium sp. strain CU-A1: evidence for naphthalene-1, 8 dicarboxylic acid metabolism. Applied and environmental microbiology, 72(9), 6034-6039. https://dx.doi.org/10.1128/AEM.00897-06

RIHS, J. D., BRENNER, D. J., WEAVER, R. E., STEIGERWALT, A. G., HOLLIS, D. G., YU, V. L. 1993. Roseomonas, a new genus associated with bacteremia and other human infections. Journal of clinical microbiology, 31(12), 3275-3283.

RÖLING, W. F., MILNER, M. G., JONES, D. M., LEE, K., DANIEL, F., SWANNELL, R. J., HEAD, I. M. 2002. Robust hydrocarbon degradation and dynamics of bacterial communities during nutrient-enhanced oil spill bioremediation. Applied and environmental microbiology, 68(11), 5537-5548. https://dx.doi.org/10.1128/AEM.68.11.5537-5548.2002

ROMINE, M. F., FREDRICKSON, J. K., LI, S. W. 1999. Induction of aromatic catabolic activity in Sphingomonas aromaticivorans strain F199. Journal of industrial microbiology \& biotechnology, 23(4), 303-313. https://dx.doi.org/10.1038/sj.jim.2900750

SAADOUN, I. 2002. Isolation and characterization of bacteria from crude petroleum oil contaminated soil and their potential to degrade diesel fuel. Journal of basic microbiology, 42(6), 420-428.

SAUL, D. J., AISLABIE, J. M., BROWN, C. E., HARRIS, L., FOGHT, J. M. 2005. Hydrocarbon contamination changes the bacterial diversity of soil from 
around Scott Base, Antarctica. FEMS Microbiology Ecology, 53(1), 141-155 https://dx.doi.org/10.1016/j.femsec.2004.11.007

SHANKAR, S., KANSRAJH, C., DINESH, M. G., SATYAN, R. S., KIRUTHIKA, S., THARANIPRIYA, A. 2014. Application of indigenous microbial consortia in bioremediation of oil-contaminated soils. International Journal of Environmental Science and Technology,11(2), 367-376. https://dx.doi.org/10.1007/s13762-013-0366-1

SHI, B. H., ARUNPAIROJANA, V., PALAKAWONG, S., YOKOTA, A. 2002 Tistrella mobilis gen. nov., sp. nov., a novel polyhydroxyalkanoate-producing bacterium belonging to $\alpha$-Proteobacteria. The Journal of general and applied microbiology, 48(6), 335-343. https://dx.doi.org/10.2323/jgam.48.335

SILVA, I. S., DOS SANTOS, E. D. C., DE MENEZES, C. R., DE FARIA, A. F. FRANCISCON, E., GROSSMAN, M., DURRANT, L. R. 2009. Bioremediation of a polyaromatic hydrocarbon contaminated soil by native soil microbiota and bioaugmentation with isolated microbial consortia. Bioresource Technology, 100(20), 4669-4675

https://dx.doi.org/10.1016/j.biortech.2009.03.079

STARKEY, R. L. 1935. Products of the oxidation of thiosulfate by bacteria in mineral media. The Journal of general physiology, 18(3), 325-349. https://dx.doi.org/10.1085/jgp.18.3.325

SUOMINEN, L., JUSSILA, M. M., MÄKELÄINEN, K., ROMANTSCHUK, M., LINDSTRÖM, K. 2000. Evaluation of the Galega-Rhizobiumgalegae system for the bioremediation of oil-contaminated soil. Environmental pollution, 107(2), 239-244. https://dx.doi.org/10.1016/S0269-7491(99)00143-8

SUZUKI, S., HIRAISHI, A. 2007. Novosphingobium naphthalenivorans sp. nov. a naphthalene-degrading bacterium isolated from polychlorinated-dioxincontaminated environments. The Journal of general and applied microbiology, 53(4), 221-228. https://dx.doi.org/10.2323/jgam.53.221

U. S. Environmental Protection Agency. (2002). Application, Performance, and Costs of Biotreatment Technologies for Contaminated Soils, EPA/600/R-03/037, Battelle Contract No.68-C-00e185.

UHLIK, O., WALD, J., STREJCEK, M., MUSILOVA, L., RIDL, J., HROUDOVA, M., ... MACEK, T. 2012. Identification of bacteria utilizing biphenyl, benzoate, and naphthalene in long-term contaminated soil. PloS one, 7(7), e40653. https://dx.doi.org/10.1371/journal.pone.0040653

WANG, J., XU, H., GUO, S. 2007. Isolation and characteristics of a microbial consortium for effectively degrading phenanthrene. Petroleum Science, 4(3), 6875. https://dx.doi.org/10.1007/s12182-007-0012-y

WANG, X., TENG, Y., LUO, Y., DICK, R. P. 2016. Biodegradation of 3, 3', 4, 4'-tetrachlorobiphenyl by Sinorhizobium meliloti NM. Bioresource technology, 201, 261-268. https://dx.doi.org/10.1016/j.biortech.2015.11.056 WEN, Y., HUANG, X., ZHOU, Y., HONG, Q., LI, S. 2011. Hansschlegelia zhihuaiae sp. nov., isolated from a polluted farmland soil. International journal of systematic and evolutionary microbiology,61(5), 1114-1117. https://dx.doi.org/10.1099/ijs.0.021543-0

XU, L., TENG, Y., LI, Z. G., NORTON, J. M., LUO, Y. M. 2010. Enhanced removal of polychlorinated biphenyls from alfalfa rhizosphere soil in a field study: the impact of a rhizobial inoculum. Science of the total environment, 408(5), 1007-1013

https://dx.doi.org/10.1016/j.scitotenv.2009.11.031

ZHANG, D. C., LIU, H. C., ZHOU, Y. G., SCHINNER, F., MARGESIN, R. 2011. Tistrella bauzanensis sp. nov., isolated from soil. International journal of systematic and evolutionary microbiology,61(9), 2227-2230. https://dx.doi.org/10.1099/ijs.0.026930-0

ZHANG, D. C., MÖRTELMAIER, C., MARGESIN, R. 2012. Characterization of the bacterial archaeal diversity in hydrocarbon-contaminated soil. Science of the Total Environment, 421, 184-196. https://dx.doi.org/10.1016/j.scitotenv.2012.01.043

ZHAO, H. P., WANG, L., REN, J. R., LI, Z., LI, M., GAO, H. W. 2008 Isolation and characterization of phenanthrene-degrading strains Sphingomonas sp. ZP1 and Tistrella sp. ZP5. Journal of hazardous materials, 152(3), 1293 1300. https://dx.doi.org/10.1016/j.jhazmat.2007.08.008

ZHENG, W., LI, D., ZHAO, J., LIU, C., ZHAO, Y., XIANG, W., WANG, X 2017. Promicromonospora soli sp. nov., a novel actinomycete isolated from soil. International journal of systematic and evolutionary microbiology, 67(10), 3829-3833. https://dx.doi.org/10.1099/ijisem.0.002207 